\title{
La gestión de la información en materia comunicativa. Una propuesta metodológica para la aplicación de las redes sociales
}

\author{
Jéssica IZQUIERDO-CASTILLO \\ Universitat Jaume I \\ jizquier@uji.es
}

Recibido: 09/11/2012

Aceptado: 23/01/2013

\begin{abstract}
Resumen
La sociedad-red evoluciona de forma constante, con la complicidad de los usuarios. En el entorno global, las redes sociales se han convertido en el espacio por excelencia de la interacción social, pero también constituyen un espacio inestimable para la permanente actualización informativa. En este artículo presentamos una propuesta para la aplicación de las redes sociales en su combinación social e informativa, que permita desarrollar nuevas formas en la gestión de la información para generar conocimiento. Los resultados aportan una metodología que combina el uso dual que los miembros de la sociedad-red realizan de las redes sociales, para aplicarlo sobre objetos de estudio cambiantes, que exigen un alto grado de actualización, como lo es el conjunto de sectores comunicativos.
\end{abstract}

Palabras clave: comunicación, redes sociales, estructura, industria cultural, audiovisual

\section{Managing the Communication in Information. A Methodological Proposal for Social Network Application}

\begin{abstract}
The network society is constantly evolving, with the complicity of the users. In the global environment, social networks have both become the preferred forum for social interaction and an invaluable space for constantly updated information. In this paper we present a proposal for the implementation of social networks as a tool that combines both social and informative dimension. It allows the development of new ways to manage information to create knowledge. The results provide a methodology that combines the dual use that network-users do with social networks. This methodology is applied on objects of study in constant change, wich demand a high level of updating, as the case of media sectors.
\end{abstract}

Keywords: Communication, Social Networks, Structure, Culture Industry, Audiovisual

Referencia normalizada

IZQUIERDO-CASTILLO, Jéssica (2013): "La gestión de la información en materia comunicativa. Una propuesta metodológica para la aplicación de las redes sociales". Estudios sobre el mensaje periodistico. Vol. 19. Núm. especial abril, págs.: 833-841. Madrid, Servicio de Publicaciones de la Universidad Complutense.

Sumario: 1. Introducción: la investigación comunicativa y la gestión de su constante actualización. 2. Metodología: el desarrollo de competencias comunicativas a través de las redes sociales. 3. El uso de redes sociales como herramienta de dinamización del debate: una propuesta práctica. 4. Conclusiones. 5. Referencias bibliográficas.

\section{Introducción: la investigación comunicativa y la gestión de su constante ac- tualización}

En el campo de investigación mediática, existe un elevado grado de dinamismo en el objeto de estudio. Los investigadores se enfrentan a una dificultad permanente, que les obliga a la constante actualización de su objeto de estudio. Esta dificultad se refleja en la docencia, donde se pone de manifiesto que no es suficiente con la exposición y 
el estudio de un momento concreto de la situación, ya que esto sólo proporciona la visión de un punto en la evolución del sistema comunicativo. Por tanto, es necesario dotar al alumnado de herramientas de gestión de la información que le permitan acceder a la actualización.

La aplicación docente de la labor investigadora en las materias que nos ocupan se realiza sobre las asignaturas vinculadas a la estructuración del sector comunicativo y a la organización de la oferta de contenidos. Estas materias están particularmente ligadas a fenómenos dinámicos de cambio, entre los que destacan la economía, la política, la tecnología, la sociedad, etc. Desde una perspectiva teórica, el/la investigador/a encuentra en el marco de la economía política de la comunicación (Mosco, 2009; Castells, 2006, 2001; Mosco, 2006; Bernardo Paniagua, 2006; Murdock y Golding, 2005; Mastrini y Sierra, 2005; Miège, 2004; Herman y McChesney, 2002; Murciano, 1992; Granham, 1985) un modelo de análisis aglutinador de las principales dimensiones relacionadas con el sistema mediático. Por otro lado, la dualidad de este sistema, que tiene la premisa en la doble naturaleza de los sectores que lo componen, dividida en economía y cultura, pone de manifiesto la necesidad de debate en el estudio de las industrias culturales. En el conjunto de estas industrias, la radio y la televisión ocupan un papel central como protagonistas de la conformación de las sociedades de la información. Los trabajos, entre otros, de Álvarez Monzoncillo (2011, 2007, 2004, 1997 , 1995), Bouquillon y Combès (2007), Zallo (2007, 1988), Bustamante (2007, 2003, 2002, 1999), Wasko (2005), Busquet (2005), Lacroix y Temblay (1997) y Flichy (1982) profundizan en su estudio. Por tanto, la estructura del sistema comunicativo, así como la organización de la oferta de contenidos de sus sectores, adquieren una gran responsabilidad en la configuración de la sociedad de la información, con una gran influencia política y social (Reig, 2009), que, en el espacio convergente, rompe las fronteras en la formación de una sociedad interconectada (sociedad red) (Castells, 2006). Todo ello adquiere mayor relevancia cuando se contempla la capacidad de los medios para actuar como aliados en la formación de la opinión pública y la construcción de la realidad social (Casero, 2008).

La materia relacionada con la estructura del sistema comunicativo, persigue desarrollar en el alumnado las competencias relacionadas con la capacidad para identificar a los actores que operan en el seno del sistema comunicativo y audiovisual. Para ello, es necesario que el alumnado conozca las características y la estructura de propiedad de los actores comunicativos, así como su evolución y las estrategias de desarrollo en el mercado. En segundo lugar, el alumnado debe adquirir la competencia para detectar nuevas opciones de mercado, así como campos de negocio novedosos, que puedan plantearse en los diferentes sectores del sistema comunicativo. En tercer lugar, deben adquirir competencia para generar reflexión crítica sobre la situación del sistema comunicativo, especialmente de sus principales actores, en relación con su contribución a la conformación de la Sociedad de la Información. Por su parte, la materia vinculada a la oferta y programación de contenido mediático, persigue el desarrollo de competencias relacionadas con la capacidad para el uso de las principales metodologías de análisis de programas y contenidos de las parrillas de programación, así como la capacidad para desarrollar diversas funciones relativas a la elaboración de las parrillas. Para ello, es necesario que el alumnado se familiarice con los contenidos 
y cambios de programación continuos que se producen en las parrillas radiofónicas y, sobre todo, televisivas, así como conocer en detalle los resultados de audiencia y sus fluctuaciones. Esto le permite reflexionar sobre la actuación de los medios y sobre las implicaciones de las prácticas profesionales en el sector comunicativo.

Por lo tanto, el debate se articula como un método de aprendizaje especialmente válido para la adquisición de competencias relacionadas con la gestión de información actualizada, la capacidad argumentativa y la reflexión crítica. La elección de las redes sociales para su articulación, permite ventajas añadidas, como la capacidad de enlazar ideas con fuentes informativas, el mantenimiento del debate en un periodo de tiempo mayor que el debate presencial, la maduración de las réplicas y la visibilidad de los comentarios en forma de publicación en Red. Este último elemento imprime mayor presión y responsabilidad en el alumnado, que se siente evaluado, no sólo por el personal docente, sino por sus compañeros, en una evaluación horizontal (García, Peñafiel y otros, 2010) que, en ocasiones, puede condicionar su compromiso con el trabajo expuesto.

En este contexto, las redes sociales constituyen una herramienta metodológica en la docencia de estudios superiores. Existen numerosos trabajos sobre la aplicación de Internet en el aprendizaje y la enseñanza, basados en las múltiples ventajas que representa el uso de esta herramienta (Espuny et al, 2011; Sierra y Sotelo, 2010; De Haro, 2010; Castañeda, 2010; Duart, 2009). Una de las principales motivaciones de estos estudios es la adecuación de los métodos docentes a la realidad social del alumnado. Los sectores más jóvenes de la sociedad presentan una elevada asimilación de Internet y los recursos de interacción que provee. Según los datos del Estudio General de Medios (EGM, 2012), la red alcanza un 70,7\% entre los jóvenes españoles de 14 a 19 años, y un 69,3\% entre los de edades comprendidas entre 20 y 24 años. Estos datos ponen de manifiesto la relevancia de Internet en el día a día de los jóvenes. Además, obligan a la docencia universitaria a plantear una incorporación de los recursos que proporciona Internet para las metodologías docentes, con el objetivo de acercarlas al universo social del alumnado. En este sentido, el Observatorio de las Redes Sociales señala que se ha producido un fenómeno de complejización de estas herramientas en Internet durante 2011. De hecho, el 91\% de los internautas dicen utilizar, al menos, una red social, de las 2,34 redes de media que tienen cada uno. Las redes que más crecieron en 2011 fueron Facebook, Twitter y LinkedIn. En su conjunto, Facebook es la que mayor penetración alcanza, con un $85 \%$ en 2011 y un incremento de 7 puntos porcentuales respecto a 2010. Le siguen Tuenti, con cierto estancamiento, en un 36\% (sólo un punto más que en 2010) y Twitter, que destaca con un aumento del doble de cuentas, pasando del 14\% al 32\% en 2011 (The Cocktail Analysis, abril 2012).

\section{Metodología: el desarrollo de competencias comunicativas a través de las redes sociales}

La metodología tiene como objeto de estudio los grupos de tercer curso de los grados de Comunicación audiovisual, de Publicidad y relaciones públicas y de Periodismo, de la Unviersitat Jaume I de Castellón. Sobre estos grupos, se aplica una metodología 
de estudio de caso que toma como punto de partida las guías docentes de las asignaturas de Estructura del sistema comunicativo y de Programación de radio y televisión, para desarrollar una propuesta de aplicación práctica del uso de la tecnología para la mejora del diseño metodológico de la asignatura.

La tecnología ofrece un amplio abanico de posibilidades como herramienta para el aprendizaje y la obtención de competencias en materia comunicativa. Esta propuesta toma el uso de las redes sociales y su aplicación para la gestión de información y la adquisición de conocimiento. Se trata de elaborar el diseño de una propuesta práctica de aprendizaje colaborativo y convergente, para lo que se articula un estudio de las herramientas sociales participativas de la web 2.0.

El objetivo es la elaboración de una propuesta práctica de aplicación de las redes sociales para la gestión y dinamización del debate virtual colaborativo. De esta forma, se proponen metodologías participativas entre los estudiantes universitarios. Para el diseño de estas metodologías, la docencia debe aprovechar el uso de herramientas comunicativas que el alumnado conoce y maneja de manera independiente. Las ventajas asociadas a las redes sociales, y a la Web 2.0 en su totalidad, son extensibles a cualquier proceso de aprendizaje. No obstante, a estas posibilidades, se suma la capacidad de las redes sociales para conocer y compartir fuentes de información, lo que supone un elemento clave para la formación en materia comunicativa, principalmente, en lo que afecta a la estructuración del sistema comunicativo y a la oferta de contenidos mediáticos, ya que una de sus principales características es la constante evolución del objeto de estudio.

\section{El uso de redes sociales como herramienta de dinamización del debate: una propuesta práctica}

La propuesta metodológica para las prácticas de las asignaturas relacionadas con la estructura del sistema comunicativo y de la programación de contenidos, está basada en el empleo de las herramientas comunicativas de la web 2.0 y 3.0. Estas herramientas permiten, de forma coordinada, el estudio del sector audiovisual y sus contenidos. En primer lugar, se establecen las bases para el uso de redes sociales aplicadas a un proceso de debate, tanto para la búsqueda de temas, como para el desarrollo y seguimiento del debate. En segundo lugar, se articulan los requisitos para transformar la información recogida de forma colaborativa en un debate a través de las redes. En tercer lugar, los esfuerzos por definir una metodología de estas características están encaminados a establecer resultados que puedan ser sometidos a evaluación y actualización por el grupo, pero también por alumnado de otros cursos.

Las redes sociales están al servicio de las relaciones entre los jóvenes, pero también facilitan la información, la crítica y el debate. El uso de estas formas de interacción permite al alumnado afianzar conocimientos a través de la participación (Sierra y Sotelo, 2010). La introducción de las redes sociales en la docencia está vinculada a modelos educativos constructivistas y colaborativos, que contribuyen a la adquisición de conocimientos, pero también a la capacidad de gestionarlos de forma duradera. Un estudio realizado en la universidad de Málaga, determina que, si bien los estudiantes únicamente se conectan a las redes sociales un $24,7 \%$ por motivos relacionados con 
los estudios, un 59,9\% considera que sería positivo crear grupos para asignaturas concretas (Gómez, Roses y Arias, 2012). Por lo tanto, parece conveniente aprovechar la predisposición del alumnado al uso de redes sociales para su aplicación académica y dotar a la docencia de propuestas metodológicas que faciliten su inclusión en los procesos de aprendizaje.

Por lo tanto, el alumnado es el encargado de adoptar una posición activa frente a un tema específico, sobre el cual debe articular un debate multimedia a través de una red social. De esta forma, la propuesta se divide en tres partes diferenciadas.

1. Propuesta consensuada de tema. En primer lugar, el alumnado consensúa, por grupos, un tema de debate relacionado con el programa de prácticas.

2. Investigación bibliográfica y debate. El alumnado procede a la búsqueda de información relacionada con el tema de debate y plantea, a través de la plataforma de red social elegida, las ideas principales de su argumentación, que se respalda por los enlaces a material informativo de referencia.

3. Procesamiento de información y extracción de conclusiones. El alumnado gestiona la información debatida en las redes sociales para extraer conclusiones razonadas y argumentadas, que se presentan redactadas con un formato susceptible de ser publicado en un blog informativo.

El resultado de esta práctica permite al alumnado de ambas materias desarrollar las competencias diseñadas para su aprendizaje práctico (tabla 1).

Tabla 1. Competencias de las enseñanzas prácticas de las materias de estructura y programación en relación a la propuesta metodológica basada en redes sociales. Fuente: elaboración propia

\begin{tabular}{|c|c|c|}
\hline MATERIA & $\begin{array}{l}\text { COMPETENCIAS ENSEÑANZAS } \\
\text { PRÁCTICAS }\end{array}$ & $\begin{array}{c}\text { PROPUESTA } \\
\text { METODOLÓGICA }\end{array}$ \\
\hline \multirow{4}{*}{ ESTRUCTURA } & $\begin{array}{l}\text { Capacidad para identificar a los actores que } \\
\text { operan en el seno del sistema comunicativo y } \\
\text { audiovisual, conociendo sus características, } \\
\text { su estructura de propiedad, sus estrategias y } \\
\text { su evolución en el mercado }\end{array}$ & Preparación del debate \\
\hline & $\begin{array}{l}\text { Competencia para detectar nuevas opciones de } \\
\text { mercado y nuevos campos de negocio en los } \\
\text { diferentes sectores del sistema comunicativo } \\
\text { y audiovisual, a partir del conocimiento deta- } \\
\text { llado de la situación de cada uno de sus ám- } \\
\text { bitos }\end{array}$ & $\begin{array}{l}\text { Búsqueda de informa- } \\
\text { ción actualizada y de- } \\
\text { fensa de un punto de } \\
\text { vista }\end{array}$ \\
\hline & $\begin{array}{l}\text { Competencia para generar reflexión crítica } \\
\text { sobre la situación del sistema comunicativo y } \\
\text { de sus principales agentes en relación con su } \\
\text { contribución a la conformación de la Socie- } \\
\text { dad de la Información }\end{array}$ & $\begin{array}{l}\text { Defensa de una postura } \\
\text { en el debate }\end{array}$ \\
\hline & $\begin{array}{l}\text { Reconocimiento a la diversidad y multicultura- } \\
\text { lidad }\end{array}$ & Debate \\
\hline \multirow{2}{*}{ PROGRAMACIÓN } & $\begin{array}{l}\text { Capacidad para el manejo de las principales } \\
\text { metodologías de análisis de programas y con- } \\
\text { tenidos de las parrillas de programación }\end{array}$ & $\begin{array}{l}\text { Búsqueda de informa- } \\
\text { ción actualizada }\end{array}$ \\
\hline & $\begin{array}{l}\text { Capacidad para llevar a cabo diversas funcio- } \\
\text { nes relativas a la elaboración de parrillas de } \\
\text { programación }\end{array}$ & $\begin{array}{l}\text { Defensa de una postura } \\
\text { en el debate }\end{array}$ \\
\hline
\end{tabular}




\begin{tabular}{|c|c|c|}
\hline \multirow{4}{*}{$\begin{array}{l}\text { ESTRUCTURA Y } \\
\text { PROGRAMACIÓN }\end{array}$} & Resolución de problemas & $\begin{array}{l}\text { Réplica en el debate. Re- } \\
\text { flexión crítica en las } \\
\text { conclusiones }\end{array}$ \\
\hline & Capacidad de análisis y síntesis & $\begin{array}{l}\text { Dinámica de debate con- } \\
\text { ciso adaptado a las exi- } \\
\text { gencias de las redes } \\
\text { sociales }\end{array}$ \\
\hline & Temas de actualidad & Información continua \\
\hline & Búsqueda individual de la información & $\begin{array}{l}\text { Preparación del debate y } \\
\text { desarrollo }\end{array}$ \\
\hline
\end{tabular}

La elección de la red social requiere la evaluación de una serie de factores. En primer lugar, se observa una tendencia general en la búsqueda de información a través de las redes sociales, con un total de $59 \%$ de internautas miembros de comunidades que giran en torno a una temática concreta, lo que marca un aumento considerable respecto a $2010(45 \%)$. Los principales temas de interés se encuentran en la informática (29\%), el ocio (28\%) y la música (27\%) (The Cocktail Analysis, 2012). En segundo lugar, se observa cierta migración de los internautas de redes sociales hacia otras plataformas más afines a sus necesidades relacionales e informativas. En este sentido destacan las motivaciones que los usuarios dan cuando cambiar de redes tradicionales y migran hacia otras plataformas. En el caso de Facebook existe poca diversidad de salidas, ya que la mayoría de internautas se debaten entre esta red y Tuenti. Sin embargo, existe un dato significativo en el abandono de las otras redes mayoritarias, como Tuenti y Twitter, con un 36\% y un 51\% respectivamente. Entre los motivos dados por los internautas, existen comentarios ligados al atractivo que supone compartir espacio de comunicación horizontal con personas relevantes en un escenario, así como por proporcionar un espacio de participación de la actualidad. Este último motivo está estrechamente vinculado al contexto televisivo, donde los iternautas han encontrado una forma de entretenimiento ligado al consumo de este medio. En tercer lugar, se observa que Twitter es la red más vinculada con la movilidad, ya que un $52 \%$ de los que se conectan diariamente lo hacen a través del móvil, mientras que los que lo hacen a Facebook o Tuenti son el 30\% y el 36\% respectivamente (The Cocktail Analysis, 2012). Esta plataforma se está consolidando con un carácter propio, vinculado más a la redistribución de información y foro de comentarios públicos, que como una forma de interacción social, más presente en otras redes. En este sentido, el $77 \%$ siguen o leen hashtags sobre algún tema concreto, mientras que el $63 \%$ lo utiliza para comentar la actualidad. El uso de esta red social para el debate en el contexto docente se perfila como una opción que integra el uso habitual de esta plataforma con los objetivos de la metodología práctica. No obstante, existen algunas limitaciones a la aplicación del debate en esta plataforma. Las más llamativas son la limitación de caracteres y la fugacidad del mensaje. No obstante, este trabajo se realiza bajo etiquetas específicas que, si bien no impiden la entrada de actores externos al grupo, sí que limitan el interés general y permiten al alumnado focalizar el uso en la actividad. Por otra parte, la limitación de espacio no debe ser un impedimento para la presentación y exposición de ideas, ya que el debate se enriquece con los enlaces a fuentes o repositorios y con las conclusiones posteriores. 
En lo referente a la evaluación, el personal docente es testigo del debate a través de la red social con su propio usuario. En principio, su función está restringida a la observación, y limita su actuación a los casos de incumplimiento de la dinámica de debate. Por lo tanto, la evaluación se aplica en tres niveles. En primer lugar, la preparación del tema, en segundo lugar el desarrollo del debate y, en tercer lugar, las conclusiones que el alumnado extrae de su trabajo.

\section{Conclusiones}

La propuesta se ha elaborado en función de las competencias diseñadas para las enseñanzas prácticas de las materias. En este sentido, se ha obtenido un resultado satisfactorio, basado en las competencias específicas de cada materia, pero, especialmente, en aquellas competencias de carácter común, que se encuentran estrechamente ligadas a la capacidad de análisis y síntesis, la búsqueda de información y el tratamiento de temas de actualidad. De igual forma, las diferentes fases de la práctica tienen un carácter multidisciplinar, pues responden a diferentes competencias específicas. Esto dota de valor a esta propuesta para su uso en el estudio de la evolución del sector audiovisual y de la oferta de sus contenidos y permite su aplicación en diferentes materias.

A través de las redes sociales, convertidas en una de las principales fuentes de información, el alumnado (y el docente/investigador) pueden acceder a datos actualizados de manera inmediata. De esta forma, la interiorización que el alumnado hace de las redes sociales en su vida cotidiana, presenta un terreno propicio para añadir a este flujo informativo la presencia de temas relacionados con las materias que exigen constante actualización.

Además, existe un gran potencial en el trabajo que realiza el alumnado, pues no sólo le permite debatir temas actuales directamente ligados a su ámbito de estudio, sino que pueden entrar en contacto con las principales fuentes, así como con los actores relacionados con el cambio. Además, a través de las redes sociales someten su trabajo al escrutinio público, no sólo de sus compañeros, sino del conjunto de internautas, entre los que también se encuentran los protagonistas de la actualidad. Estas características dotan a la práctica en redes sociales de una dimensión de participación imposible de alcanzar en el aula y posibilita la integración de la vida personal del alumnado con su ámbito de aprendizaje y con el contexto profesional.

\section{Referencias bibliográficas}

ÁLVAREZ MONZONCILLO, José María (2011): La televisión etiquetada: nuevas audiencias, nuevos negocios. Madrid, Ariel.

ÁLVAREZ MONZONCILLO, José María (2004): El futuro del ocio en el hogar. Madrid, Fundación autor.

ÁLVAREZ MONZONCILLO, José María (1997): Imágenes de pago. Madrid, Fragua. ÁLVAREZ MONZONCILLO, José María (1995): El cine en la era del audiovisual. Madrid, Cátedra. 
ÁLVAREZ MONZONCILLO et al. (2007): Alternativas de politica cultural. Las industrias culturales en las redes digitales (disco, cine libre, derechos de autor). Madrid, Gedisa.

ASOCIACIÓN PARA LA INVESTIGACIÓN DE MEDIOS DE COMUNICACIÓN (AIMC) (2011): Navegantes en la Red. Encuesta AIMC a usuarios de Internet. Octubre-diciembre 2011. Disponible en http://www.aimc.es. [Consultado: 24 de septiembre de 2012]

BERNARDO, Jose María (2006): El sistema de la comunicación mediática. De la comunicación interpersonal a la comunicación global. Valencia, Tirant lo Blanch.

BUSTAMANTE, Enrique (1999): La televisión económica. Financiación, estrategias y mercados. Barcelona, Gedisa.

BUSTAMANTE, Enrique (coord., 2002): Comunicación y cultura en la era digital. Industrias, mercados y diversidad en España. Barcelona, Gedisa.

BUSTAMANTE, Enrique (coord., 2003): Hacia un sistema mundial de comunicación. Las industrias culturales en la era digital. Barcelona, Gedisa.

CASERO, Andreu (2008): La construcción mediática de las crisis políticas. Madrid, Fragua.

CASTAÑEDA, Linda. (2010). Aprendizaje con redes sociales. Tejidos educativos para los nuevos entornos. Sevilla, MAD.

CASTELLS, Manuel (ed., 2006): La sociedad red: una visión global. Madrid, Alianza.

CASTELLS, Manuel (2001): La galaxia Internet. Barcelona, Plaza\&Janés.

DE HARO, Juan José (2010): Redes sociales para la educación. Madrid, Anaya.

DUART, Josep M. (2009): "Internet, redes sociales y educación”. Revista Universidad y Sociedad del Conocimiento, 6, 1

ESPUNY, Cinta; GONZÁLEZ, Juan; LLEIXA FORTUÑO, Mar; y GISBERT CERVERA, Mercè (2011): “Actitudes y expectativas del uso educativo de las redes sociales en los alumnos universitarios". Revista de Universidad y Sociedad del Conocimiento, 8, 1, pp. 171-185.

GARCÍA, Daniel; PEÑAFIEL, Carmen; y otros (2010): “¿Y si me suspenden mism propios compañeros? Aportación de las tic a la interconectividad transversal entre asignaturas de periodismo como herramienta legitimadora del proceso de evaluación”, en SIERRA SÁNCHEZ, Javier y SOTELO GONZÁLEZ, Joaquín: Métodos de innovación docente aplicados a los estudios de Ciencias de la Comunicación. Madrid, Fragua.

GÓMEZ, Marisol; ROSES, Sergio y FARIAS, Pedro (2011): "El uso académico de las redes sociales en universitarios". Comunicar, 38, v. XIX.

HERMAN, Edward S.; McCHESNEY, Robert W. (1997): Los medios globales. Los nuevos misioneros del capitalismo corporativo. Madrid, Cátedra.

MOSCO, Vicent (2009): The Political Economy of Communication. London, Sage. 
MURCIANO, Marcial (1992): Estructura dinámica de la comunicación internacional, Barcelona, Bosch.

OBSERVATORIO DE LAS REDES SOCIALES (2012): The Cocktail Analysis, abril 2012. Disponible en http://tcanalysis.com. [Consultado: 24 de septiembre de 2012]

REIG, Ramón (2009): "Bases teóricas y documentales para el estudio de la Estructura de la Información y el análisis estructural de los mensajes". Estudios sobre el mensaje periodístico, $\mathrm{n}^{\circ} 15$. Madrid, Servicio de Publicaciones de la Universidad Complutense, pp. 385-407.

SIERRA, Javier. y SOTELO, Joaquín. (coords., 2010): Métodos de innovación docente aplicados a los estudios de Ciencias de la comunicación. Madrid, Fragua.

WASKO, Janet (2003): How Hollywood Works. London, SAGE.

WOLF, Mauro (1989): "Géneros y televisión”. Anàlisi: quaderns de comunicación $i$ cultura, $\mathrm{n}^{\circ}$ 9. Disponible en http://www.cac.cat. [Consultado: 24 de septiembre de 2012]

ZALLO, Ramón (2007): "La economía de la cultura (y de la comunicación) como objeto de estudio" en Revista Zer, ${ }^{\circ}$ 22. Disponible en: http://www.ehu.es /zer/es/hemeroteca/articulo/la-economia-de-la-cultura-y-de-la-comunicacioncomo-objeto-de-estudio/315. Consultado el 24 de septiembre de 2012.

\section{Jéssica IZQUIERDO-CASTILLO}

Universitat Jaume I de Castellón

Facultat de Ciències Humanes i Sociales. Departament de Ciències de la Comunicació Profesora Adjunta Doctora jizquier@uji.es 